\title{
Molecular analysis of Phytophthora species found in Poland
}

\author{
Tomasz Oszako ${ }^{1}$, Katarzyna Sikora ${ }^{2}$, Lassaâd Belbahri $^{3}$, Justyna A. Nowakowska ${ }^{4}$ \\ ${ }^{1}$ Bialystok University of Technology, Faculty of Forestry in Hajnówka, Piłsudskiego 8, 17-200 Hajnówka, Poland, \\ e-mail: t.oszako@pb.edu.pl \\ ${ }^{2}$ Forest Research Institute, Department of Forest Protection, Sękocin Stary, Braci Leśnej 3, 05-090 Raszyn, Poland \\ ${ }^{3}$ University of Neuchâtel, Laboratory of Soil Biology, Rue Emile Argand 11, 2009 Neuchâtel, Switzerland \\ ${ }^{4}$ Cardinal Stefan Wyszyński University in Warsaw, Faculty of Biology and Environmental Sciences, Wóycickiego 1/3, \\ 01-938 Warsaw, Poland
}

\section{Abstract}

Pathogens of Phytophthora genus are common not only in forest nurseries and stands, but also in water courses. Species of Phytophthora spread with plants for plantings (and soil attached to them) and with water courses as well, attacking the plants growing in riparian ecosystems. Several specialized organisms damaging only one tree species were identified like P. alni on alders or P. quercina on oaks. Some Phytophthora species can develop on several hosts like $P$. plurivora and $P$. cactorum on oaks, beeches, alders, ashes and horse chestnuts. Other oomycetes like $P$. gallica species was found for the first time in Poland in water used for plant watering in forest nursery. Species $P$. lacustris and $P$. gonapodyides were found in superficial water. Phytophthora species $P$. polonica was identified in the declining alder stands for the first time in the world, and $P$. taxon hungarica and $P$. megasperma were found in the rhizosphere of seriously damaged ash stands for the first time in Poland. The most often isolated species were $P$. plurivora (clade 2) with frequency $37 \%$ and $P$. lacustris with frequency $33 \%$ (clade 6). The best represented clade 6 revealed the occurrence of 6 species: $P$. gonapodyides, P. lacustris, P. megasperma, $P$. sp. raspberry, $P$. taxon hungarica and $P$. taxon oak soil.

\section{KeY WORDS}

fine root pathogens, sequencing DNA, alien, invasive, emerging

\section{INTRODUCTION}

An increase in trade of plants and globalization pose a risk of plant disease epidemics, resulting from introductions of exotic plant pathogens. An associated risk that accelerates pathogen evolution may occur as a consequence of genetic exchange between introduced or introduced and resident pathogens (Brasier et al. 1999).
There is a likelihood of such evolutionary events occurring in Poland, as well. On the other hand, new diagnostic methods based on molecular tools are currently sufficiently sensitive to allow detection of new phytopathogens. Recently, in forestry, emerging diseases are caused by invasive, alien oomycetes, which are soil-borne fine root pathogens, sometimes specializing in damage of certain forest tree species as their host. As established in 
Central Europe, Phytophthora quercina is often recovered from declining oaks proved to be more pathogenic to European oaks Q. robur than any other Phytophthora species (Jung et al. 1999). The common species Phytophthora plurivora occurs all over Italy, while $P$. quercina is the species significantly associated with declining of oak trees (Vettraino et al. 2002). In Italy, eleven soil-borne species of Phytophthora were detected in oak forests with $35 \%$ as the frequency of isolations, being also correlated with soil $\mathrm{pH}$ and longitude of the sites. P. cactorum was recovered from sites in central and southern Italy, whereas $P$. quercina was isolated in the northern and central part of the country. In Denmark, several species of Phytophthora were found in the rhizosphere of declining ash trees (Orlikowski et al. 2011); earlier, they were also found in nurseries (Jung et al. 2016).

Since pathogens from genus Phytophthora are responsible for serious diseases world-wide and can occur on a wide range of hosts, in the present study, we concentrated on an assessment of the occurrence of these pathogens in the Polish forest nurseries and stands.

\section{MAterial AND MEthodS}

Soil, together with the root system, was sampled in plastic bags weighing $0.5 \mathrm{~kg}$ each and isolation tests were performed using rhododendron, oak or beech leaves as baits. Water was collected with 1.51 plastic bottles, which were sterilized with $70 \%$ ethanol and washed with distilled water. The sampled water was filtered in the lab, using the Millipore vacuum pump with nylon filters of $5 \mu \mathrm{m}$ pore-size. Filters with biological sediment as well as the fragments of discoloured bait tissues were placed on selective PARPNH medium (potato dextrose agar amended with $10 \mu \mathrm{g} \mathrm{ml}^{-1}$ pimaricin, $200 \mu \mathrm{g} \mathrm{ml}^{-1}$ ampicillin, $10 \mu \mathrm{g} \mathrm{ml}^{-1}$ rifampicin, $25 \mu \mathrm{g} \mathrm{ml}^{-1}$ pentachloronitrobenzene (PCNB), $50 \mu \mathrm{g} \mathrm{ml}^{-1}$ nystatin, and $50 \mu \mathrm{g} \mathrm{ml}^{-1}$ hymexazol).

Pure cultures of Phytophthora sp. isolates obtained from the water and soil samples were grown in the liquid V8 media (100 ml clarified V8 juice in $900 \mathrm{ml}$ distilled water, amended with $2 \mathrm{~g}$ of $\mathrm{CaCO}_{3}$ for 3-5 days in the dark at $22-25^{\circ} \mathrm{C}$. The mycelium was subsequently rinsed in sterile distilled water, dried and disrupted in liquid nitrogen prior to the DNA extraction. Total DNA was extracted from mycelium by using GenElute ${ }^{\mathrm{TM}}$ Plant
Genomic DNA Miniprep Kit (Sigma-Aldrich ${ }^{\circledR} \mathrm{GmbH}$, Germany), following the manufacturer's protocol. Polymerase chain reaction (PCR) amplification of the ITS region of the template DNA was performed using primers ITS6 and ITS4 (White et al. 1990; Cooke et al. 2000) in a $50 \mu 1$ reaction containing $50-100 \mathrm{ng}$ genomic DNA, $250 \mathrm{nM}$ of each primer, $200 \mu \mathrm{M}$ of each dNTP, $1 \mathrm{mM}$ $\mathrm{MgCl}_{2}$, 1U Taq polymerase, 1xQ solution and 1xPCR buffer (Qiagen Ltd., Valencia, CA, USA). The reaction was performed in a PTC-200Ô Programmable Thermal Controller (MJ Research, Inc.) for 40 cycles of denaturation at $94^{\circ} \mathrm{C}$ for $30 \mathrm{~s}$, annealing at $55^{\circ} \mathrm{C}$ for $30 \mathrm{~s}$ and extension at $72^{\circ} \mathrm{C}$ for $50 \mathrm{~s}$, with initial denaturation of 3 min at $94^{\circ} \mathrm{C}$ before cycling and a final extension of $10 \mathrm{~min}$ at $72^{\circ} \mathrm{C}$ after cycling. The PCR product was purified using the Clean-up kit (A\&A Biotechnology), following the manufacturer's protocol. Sequencing was conducted on a CEQ ${ }^{\mathrm{TM}} 8000$ 9.0.25 automated sequencer, (Beckman Coulter ${ }^{\circledR}$, Fullerton, USA). Forward and reverse sequences were linked in BioEdit software and the resulting sequences were aligned with NCBI Nucleotide collection.

All the collected sequences were compared in ITS1 region by using the ClustalW algorithm provided in the BioEdit software; further phylogenetic analysis was performed using MEGA5. The Maximum Likelihood method based on the Tamura-Nei model was used. The percentage of replicate trees in which the associated taxa clustered together in the bootstrap test (1000 replicates) are shown next to the branches. Initial trees for the heuristic search were obtained automatically by applying Neighbor-Joining and BIONJ algorithms to a matrix of pairwise distances estimated using the Maximum Composite Likelihood (MCL) approach, and then selecting the topology with superior log likelihood value. The tree is drawn to scale, with branch lengths measured in the number of substitutions per site. The analysis involved 117 nucleotide sequences (116 Phytophthora sequences and Pythium sterilum JX271797 sequence as an outgroup).

\section{Results}

As given in the table below, the Phytophthora isolates were identified to species on the basis of sequence alignment with NCBI database (Tab. 1). Among the list 
of identified Phytophthora isolates, there is a new species in Poland - Phytophthora gallica, which is considered to be moderately aggressive to Alnus glutinosa and Fagus sylvatica, weakly aggressive to Quercus robur and Salix alba and non-pathogenic to Fraxinus excelsior (Jung and Nechwatal 2008). The origin of $P$. gallica and its ecological role in wet ecosystems remain unclear.

Table 1. List of Phytophthora isolates used in the study

\begin{tabular}{|c|c|c|c|c|c|c|}
\hline NCBI № & Isolate & Country & Location & Host & Sample & IBL № \\
\hline 1 & 2 & 3 & 4 & 5 & 6 & 7 \\
\hline JX276034 & P. alni & Poland & Sękocin & Alnus glutinosa & forest & IBL/2011/1/1 \\
\hline JX276035 & P. alni & Poland & Sękocin & Alnus glutinosa & forest & IBL/2011/2/1 \\
\hline EF152518 & P. alni & Poland & Żyrardów & Alnus glutinosa & forest & $825 \mathrm{a}$ \\
\hline EF152517 & P. alni & Poland & Żyrardów & Alnus glutinosa & forest & $825 b$ \\
\hline EF152516 & P. alni & Poland & Żyrardów & Alnus glutinosa & forest & $825 \mathrm{c}$ \\
\hline JX276022 & P. cactorum & Poland & Konstantynowo & Fraxinus excelsior & forest & IBL/2011/212 \\
\hline JX276028 & P. cactorum & Poland & Konstantynowo & Quercus robur & forest & IBL/2011/220 \\
\hline JX276029 & P. cactorum & Poland & Konstantynowo & Fraxinus excelsior & forest & IBL/2011/221 \\
\hline JX276030 & P. cactorum & Poland & Konstantynowo & Quercus robur & forest & IBL/2011/223 \\
\hline JX276031 & P. cactorum & Poland & Konstantynowo & Fraxinus excelsior & forest & IBL/2011/225 \\
\hline JX276019 & P. cactorum & Poland & Krotoszyn & Quercus robur & forest & IBL/2011/210 \\
\hline EU240056 & P. cactorum & Poland & Moszczanka & Fagus sylvatica & forest & 798 \\
\hline EU240060 & P. cactorum & Poland & Radziejowice & Aesculus hippocastanum & park & 813 \\
\hline EU240045 & P. cactorum & Poland & Wilanowice & Fraxinus excelsior & nursery & 748 \\
\hline EU240182 & P. cactorum & Poland & Wilanowice & Quercus robur & forest & 753 \\
\hline EU240061 & P. cactorum & Poland & Wilanowice & Fagus sylvatica & forest & $764 \mathrm{~A}$ \\
\hline JX271803 & P. gallica & Poland & Kiejsze & riparian area & water & IBL/2011/28/2 \\
\hline JX276033 & P. gonapodyides & Poland & Chojnów & Alnus glutinosa & forest & IBL/2011/232 \\
\hline JX276038 & P. gonapodyides & Poland & Oborniki & Quercus robur & forest & IBL/2011/10/4/1 \\
\hline JX276041 & P. gonapodyides & Poland & Oborniki & Quercus robur & forest & IBL/2011/8/1/10 \\
\hline JX276036 & P. gonapodyides & Poland & Oborniki & Quercus robur & forest & IBL/2011/8/1/6 \\
\hline EU240125 & P. lacustris & Poland & Bug river & Quercus robur & water & WD40A \\
\hline EU240126 & P. lacustris & Poland & Bug river & Quercus robur & water & WD40C \\
\hline EU240137 & P. lacustris & Poland & Bug river & Quercus robur & water & WD40E \\
\hline EU240042 & P. lacustris & Poland & Bug river & Quercus robur & water & WD41a \\
\hline EU240175 & P. lacustris & Poland & Bug river & Quercus robur & water & WD43A \\
\hline EU240152 & P. lacustris & Poland & Chojnów & Quercus robur & nursery & GD15A \\
\hline EU240088 & P. lacustris & Poland & Dąbie & Alnus glutinosa & forest & GD7B \\
\hline EU240089 & P. lacustris & Poland & Dąbie & Alnus glutinosa & forest & GD7C \\
\hline EU240091 & P. lacustris & Poland & Dąbie & Alnus glutinosa & forest & GD7G \\
\hline EU240166 & P. lacustris & Poland & Kanał Królewski & Alnus glutinosa & water & WD47A \\
\hline EU240179 & P. lacustris & Poland & Kiejsze & riparian area & water & 920 \\
\hline EU240180 & P. lacustris & Poland & Kiejsze & riparian area & water & 921 \\
\hline EU240181 & P. lacustris & Poland & Kiejsze & riparian area & water & 922 \\
\hline
\end{tabular}




\begin{tabular}{|c|c|c|c|c|c|c|}
\hline 1 & 2 & 3 & 4 & 5 & 6 & 7 \\
\hline EU240197 & P. lacustris & Poland & Kiejsze & riparian area & water & 923 \\
\hline EU240159 & P. lacustris & Poland & Narew river & riparian area & water & WD37A \\
\hline EU240099 & P. lacustris & Poland & Narew river & riparian area & water & WD37B \\
\hline EU240160 & P. lacustris & Poland & Narew river & riparian area & water & WD37C \\
\hline EU240123 & P. lacustris & Poland & Narew river & riparian area & water & WD38A \\
\hline EU240124 & P. lacustris & Poland & Narew river & riparian area & water & WD38B \\
\hline EU240100 & P. lacustris & Poland & Narew river & riparian area & water & WD39A \\
\hline EU240161 & P. lacustris & Poland & Narew river & riparian area & water & WD39B \\
\hline JX271790 & P. lacustris & Poland & Orlanka river & riparian area & water & IBL/2011/10/1 \\
\hline JX271791 & P. lacustris & Poland & Orlanka river & riparian area & water & IBL/2011/10/2 \\
\hline EU240153 & P. lacustris & Poland & Pomiechówek & Acer pseudoplatanus & forest & GD18G \\
\hline EU240138 & P. lacustris & Poland & Rządza river & Acer pseudoplatanus & water & WD44B \\
\hline EU240164 & P. lacustris & Poland & Rządza river & Acer pseudoplatanus & water & WD44C \\
\hline EU240177 & P. lacustris & Poland & Rządza river & Acer pseudoplatanus & water & WD45A \\
\hline EU240101 & P. lacustris & Poland & Rządza river & Acer pseudoplatanus & water & WD45B \\
\hline EU240165 & P. lacustris & Poland & Rządza river & Acer pseudoplatanus & water & WD45C \\
\hline EU240167 & P. lacustris & Poland & Sękocin & Acer pseudoplatanus & water & B02 \\
\hline EU240094 & P. lacustris & Poland & Sękocin & Acer pseudoplatanus & water & B04 \\
\hline EU240037 & P. lacustris & Poland & Sękocin & Acer pseudoplatanus & water & B14 \\
\hline EU240102 & P. lacustris & Poland & Sokołówka & Quercus robur & nursery & GD36D \\
\hline EU240067 & P. lacustris & Poland & Sokołówka & Quercus robur & nursery & GD36F \\
\hline EU240066 & P. lacustris & Poland & Sokołówka & Quercus robur & nursery & GD40A \\
\hline EU240184 & P. lacustris & Poland & Sokołówka & Quercus robur & nursery & GD40C \\
\hline EU240065 & P. lacustris & Poland & Sokołówka & Quercus robur & nursery & GD40D \\
\hline JX271796 & P. lacustris & Poland & Zuzela & Quercus robur & water & IBL/2011/13/2 \\
\hline JX274423 & P. megasperma & Poland & Wolica & Fraxinus excelsior & forest & IBL/2012/9/3 \\
\hline EU240052 & P. plurivora & Poland & Biechów & Fraxinus excelsior & forest & 785 \\
\hline EU240053 & P. plurivora & Poland & Biechów & Fraxinus excelsior & forest & 786 \\
\hline EF152519 & P. plurivora & Poland & Biernatów & Quercus robur & forest & $790 \mathrm{a}$ \\
\hline EU240075 & P. plurivora & Poland & Biernatów & Quercus robur & forest & $791 \mathrm{~A}$ \\
\hline $\mathrm{JX} 276018$ & P. plurivora & Poland & Broniszew & Pyrus & orchard & IBL/2011/206 \\
\hline EU240085 & P. plurivora & Poland & Buków & Pyrus & nursery & $754 \mathrm{~A}$ \\
\hline EU240054 & P. plurivora & Poland & Dębowiec & Pyrus & forest & 788 \\
\hline EU240077 & P. plurivora & Poland & Dębowiec & Pyrus & forest & $787 \mathrm{~A}$ \\
\hline EU240076 & P. plurivora & Poland & Dębowiec & Pyrus & forest & 787B \\
\hline JX276023 & P. plurivora & Poland & Konstantynowo & Fraxinus excelsior & forest & IBL/2011/213 \\
\hline JX276024 & P. plurivora & Poland & Konstantynowo & Fraxinus excelsior & forest & IBL/2011/214 \\
\hline JX276025 & P. plurivora & Poland & Konstantynowo & Quercus robur & forest & IBL/2011/216 \\
\hline JX276027 & P. plurivora & Poland & Konstantynowo & Quercus robur & forest & IBL/2011/219 \\
\hline EU240188 & P. plurivora & Poland & Korczew & Alnus glutinosa & forest & $6 " \mathrm{a}$ \\
\hline EU240189 & P. plurivora & Poland & Korczew & Alnus glutinosa & forest & 6"b \\
\hline
\end{tabular}




\begin{tabular}{|c|c|c|c|c|c|c|}
\hline 1 & 2 & 3 & 4 & 5 & 6 & 7 \\
\hline EU240192 & P. plurivora & Poland & Korczew & Alnus glutinosa & forest & 6"”c \\
\hline EU240193 & P. plurivora & Poland & Korczew & Alnus glutinosa & forest & 6"'d \\
\hline EU240194 & P. plurivora & Poland & Korczew & Alnus glutinosa & forest & 6"”e \\
\hline EU240195 & P. plurivora & Poland & Korczew & Alnus glutinosa & forest & 6"”f \\
\hline EU240055 & P. plurivora & Poland & Lipowa & Alnus glutinosa & forest & 794 \\
\hline EU240057 & P. plurivora & Poland & Moszczanka & Fagus sylvatica & forest & 801 \\
\hline EU240050 & P. plurivora & Poland & Opawice & Fagus sylvatica & forest & 776 \\
\hline EU240051 & P. plurivora & Poland & Opawice & Quercus robur & nursery & 778 \\
\hline EU240080 & P. plurivora & Poland & Opawice & Quercus robur & nursery & $777 \mathrm{~A}$ \\
\hline EU240079 & P. plurivora & Poland & Opawice & Fagus sylvatica & nursery & 779A \\
\hline EU240078 & P. plurivora & Poland & Opawice & Fraxinus excelsior & nursery & $780 \mathrm{~A}$ \\
\hline EU240183 & P. plurivora & Poland & Pokrzywna & Fraxinus excelsior & forest & 772 \\
\hline EU240187 & P. plurivora & Poland & Pokrzywna & Fraxinus excelsior & forest & 769A \\
\hline EU240058 & P. plurivora & Poland & Szklary & Fraxinus excelsior & forest & 804 \\
\hline EU240059 & P. plurivora & Poland & Szklary & Fraxinus excelsior & forest & 806 \\
\hline EU240044 & P. plurivora & Poland & Wilanowice & Fagus sylvatica & nursery & 747 \\
\hline EU240046 & P. plurivora & Poland & Wilanowice & Fagus sylvatica & forest & 755 \\
\hline JX274421 & P. plurivora & Poland & Wolica & Fraxinus excelsior & forest & IBL/2012/3/4 \\
\hline JX274427 & P. plurivora & Poland & Wolica & Fraxinus excelsior & forest & IBL/2012/5/3 \\
\hline JX274422 & P. plurivora & Poland & Wolica & Fraxinus excelsior & forest & IBL/2012/5/5 \\
\hline JX274420 & P. plurivora & Poland & Wolica & Fraxinus excelsior & forest & IBL/2012/6/2 \\
\hline JX274425 & P. plurivora & Poland & Wolica & Fraxinus excelsior & forest & IBL/2012/7A \\
\hline JX274426 & P. plurivora & Poland & Wolica & Fraxinus excelsior & forest & IBL/2012/7c \\
\hline JX274424 & P. plurivora & Poland & Wolica & Fraxinus excelsior & forest & IBL/2012/8/1 \\
\hline JX276032 & P. plurivora & Poland & Zabuże & Alnus glutinosa & forest & IBL/2011/231 \\
\hline JX276057 & P. plurivora & Poland & Zuzela & Alnus glutinosa & water & IBL/2011/11 \\
\hline JX276051 & P. plurivora & Poland & Zuzela & Alnus glutinosa & water & IBL/2011/1/2 \\
\hline JX276052 & P. plurivora & Poland & Zuzela & Alnus glutinosa & water & IBL/2011/2/2 \\
\hline EU240063 & P. polonica & Poland & Dąbie & Alnus glutinosa & forest & GD7A \\
\hline EU240198 & P. polonica & Poland & Dąbie & Alnus glutinosa & forest & GD7D \\
\hline EU240093 & P. polonica & Poland & Dąbie & Alnus glutinosa & forest & GD7I \\
\hline JX276017 & P. polonica & Poland & Kwidzyń & Alnus glutinosa & forest & IBL/2011/204 \\
\hline JX276020 & P. quercina & Poland & Piaski & Quercus robur & forest & IBL/2011/211/1 \\
\hline JX276021 & P. quercina & Poland & Piaski & Quercus robur & forest & IBL/2011/211/2 \\
\hline EU240068 & P. sp. raspberry & Poland & Dobieszyn & Quercus petraea & nursery & GD23B \\
\hline JX274428 & P. taxon hungarica & Poland & Wolica & Fraxinus excelsior & forest & IBL/2012/9/7 \\
\hline JX274429 & P. taxon hungarica & Poland & Wolica & Fraxinus excelsior & forest & IBL/2012/9/8 \\
\hline JX276040 & P. taxon oaksoil & Poland & Oborniki & Quercus robur & forest & IBL/2011/6/1/7 \\
\hline JX276042 & P. taxon oaksoil & Poland & Oborniki & Quercus robur & forest & IBL/2011/7/1/4 \\
\hline JX276043 & P. taxon oaksoil & Poland & Oborniki & Quercus robur & forest & IBL/2011/7/1/5 \\
\hline JX276037 & P. taxon oaksoil & Poland & Oborniki & Quercus robur & forest & IBL/2011/9/1/2 \\
\hline
\end{tabular}




\section{OPEN}

As many as 12 species of Phytophthora belonging to 7 different clades were found based on the sequence analysis (Tab. 2). The most abundant clades present in Poland are clade 6 (43.1\%) and 2 (37.1\%). Phytophtho$r a$ species in clade 6 have non-papillate sporangia and are mostly infectious to roots or present in the rhizosphere. Phytophthora plurivora, the only representative of clade 2, is considered to be the cause of several devastating declines and diebacks of major forest tree species.

Table 2. Phytophthora species found in Poland

\begin{tabular}{|l|c|r|r|}
\hline \multicolumn{1}{|c|}{ Species } & Clade & $\mathrm{N}$ & $\%$ \\
\hline P. alni & $7 \mathrm{a}$ & 5 & 4.31 \\
\hline P. cactorum & $1 \mathrm{a}$ & 11 & 9.48 \\
\hline P. gallica & 10 & 1 & 0.86 \\
\hline P. gonapodyides & 6 & 4 & 3.45 \\
\hline P. lacustris & 6 & 38 & 32.76 \\
\hline P. megasperma & 6 & 1 & 0.86 \\
\hline P. plurivora & 2 & 43 & 37.07 \\
\hline P. polonica & 9 & 4 & 3.45 \\
\hline P. quercina & 4 & 2 & 1.72 \\
\hline P. sp. raspberry & 6 & 1 & 0.86 \\
\hline P. taxon hungarica & 6 & 2 & 1.72 \\
\hline P. taxon oaksoil & 6 & 4 & 3.45 \\
\hline
\end{tabular}

As shown, many of the discovered Phytophthora species were found on different hosts, including important forest tree species (Tab. 3). Also, there was a diversity in the age of host species - Phytophthora species were found in mature forest stands and on tree seedlings in forest nurseries. The identification of species like $P$. gallica, $P$. lacustris, $P$. gonapodyides and $P$. alni in riparian ecosystems was possible due to the use of water filtration techniques, plating and DNA (ITS) analysis.

Since 2000, an increasing decline and dieback of alders has been observed in Poland. Ten different species of obtained Phytophthora isolates, including those shown in Table 3, originated from diseased trunks and from rhizosphere (Trzewik et al. 2015). Phylogeny of Polish Phytophthora species is shown on the dendrogram created based on Maximum Likelihood method (Fig. 1). The new for knowledge oomycete species
P. polonica was found in declining alder stands along the river Ner (Belbahri et al. 2006).

Table 3. Host range of Phytophthora species fund in Poland

\begin{tabular}{|c|c|}
\hline Species & Hosts \\
\hline P. alni & Alnus glutinosa \\
\hline \multirow{4}{*}{ P. cactorum } & Aesculus hippocastanum \\
\hline & Fagus sylvatica \\
\hline & Fraxinus excelsior \\
\hline & Quercus robur \\
\hline \multirow{2}{*}{ P. gonapodyides } & Alnus glutinosa \\
\hline & Quercus robur \\
\hline \multirow{3}{*}{ P. lacustris } & Acer pseudoplatanus \\
\hline & Alnus glutinosa \\
\hline & Quercus robur \\
\hline P. megasperma & Fraxinus excelsior \\
\hline \multirow{5}{*}{$P$. plurivora } & Alnus glutinosa \\
\hline & Fagus sylvatica \\
\hline & Fraxinus excelsior \\
\hline & Pyrus sp. \\
\hline & Quercus robur \\
\hline P. polonica & Alnus glutinosa \\
\hline P. quercina & Quercus robur \\
\hline P. sp. raspberry & Quercus petraea \\
\hline P. taxon hungarica & Fraxinus excelsior \\
\hline P. taxon oaksoil & Quercus robur \\
\hline
\end{tabular}

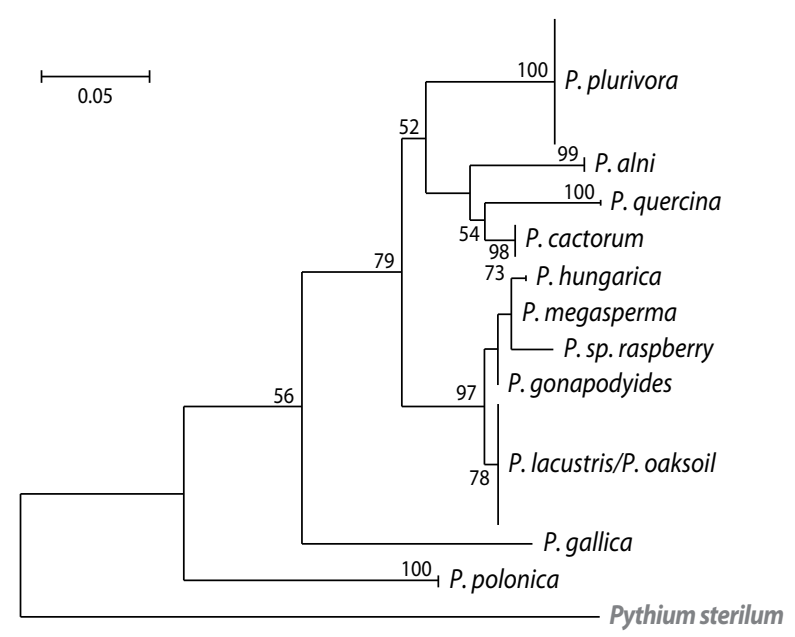

Figure 1. Phylogeny of Phytophthora isolates (ML method, bootstrap $=1000$ ) 


\section{Conclusions}

1. Pathogens of Phytophthora genus are common not only in nurseries and forest stands, but also in parks and orchards.

2. Species of Phytophthora spread with plants for plantings (and soil attached to them) and with water along water courses as well, attacking the plant associations or shelterbelts of the riparian ecosystems, especially alders.

3. Several specialized organisms damaging only one tree species were identified like $P$. alni on alders or P. quercina on oaks.

4. Some Phytophthora species can develop on several hosts like P. plurivora and P. cactorum on oaks, beeches, alders, ashes and horse chestnuts.

5. Other oomycetes like $P$. gallica species was found for the first time in Poland in water used for plant watering in the Kiejsze nursery (Koło Forest District).

6. In water ecosystems, species like P. lacustris and $P$. gonapodyides were found. The pathogenicity of these species is not fully recognized yet.

7. For the first time in the world, the new Phytophthora species $P$. polonica was identified in the declining alder stands (Koło FD); and for the first time in Poland, two other species $P$. taxon hungarica and $P$. megasperma were found in the rhizosphere of seriously damaged ash stands (showing ash dieback).

8. The most often isolated species were P. plurivora (clade 2) with frequency $37 \%$ and $P$. lacustris with frequency $33 \%$ (clade 6 ).

9. The best represented clade 6 revealed the occurrence of 6 species: P. gonapodyides, P. lacustris, $P$. megasperma, $P$. sp. raspberry, $P$. taxon hungarica and $P$. taxon oak soil.

\section{References}

Belbahri L., Moralejo E., Calmin G., Oszako T., García J.A., Descals E., Lefort F. 2006. Phytophthora polonica, a new species isolated from declining Alnus glutinosa stands in Poland. FEMS Microbiology Letters, 261 (2), 165-174.

Brasier C.M., Cooke D.E.L., Duncan J.M. 1999. Origin of a new Phytophthora pathogen through interspe- cific hybridization. Proceedings of the National Academy of Sciences, 96 (10), 5878-5883.

Cooke D.E.L., Duncan J.M., Williams N.A., Weerdt M., Bonants P.J.M. 2000. Identification of Phytophthora species on the basis of restriction enzyme fragment analysis of the internal transcribed spacer regions of ribosomal RNA. EPPO Bulletin, 30 (3/4), 519-523.

Jung T., Cooke D.E.L., Blaschke H., Duncan J.M., Osswald W. 1999. Phytophthora quercina sp. nov., causing root rot of European oaks. Mycological Research, 103 (7), 785-798.

Jung T., Nechwatal J. 2008. Phytophthora gallica sp. nov., a new species from rhizosphere soil of declining oak and reed stands in France and Germany. Mycological research, 112 (10), 1195-1205.

Jung T., Orlikowski L., Henricot B., Abad-Campos P., Aday A.G., Aguín Casal O., Bakonyi J., Cacciola S.O., Cech T., Chavarriaga D., Corcobado T., Cravador A., Decourcelle T., Denton G., Diamandis S., Doğmuş-Lehtijärvi H.T., Franceschini A., Ginetti B., Green S., Glavendekić M., Hantula J., Hartmann G., Herrero M., Ivic D., Horta Jung M., Lilja A., Keca N., Kramarets V., Lyubenova A., Machado H., Magnano di San Lio G., Mansilla Vázquez P. J., Marçais B., Matsiakh I., Milenkovic I., Moricca S., Nagy Z.Á., Nechwatal J., Olsson C., Oszako T., Pane A., Paplomatas E.J., Pintos Varela C., Prospero S., Rial Martínez C., Rigling D., Robin C., Rytkönen A., Sánchez M.E., Sanz Ros A.V., Scanu B., Schlenzig A., Schumacher J., Slavov S., Solla A., Sousa E., Stenlid J., Talgø V., Tomic Z., Tsopelas P., Vannini A., Vettraino A.M., Wenneker M., Woodward S., Peréz-Sierra A. 2016. Widespread Phytophthora infestations in European nurseries put forest, semi-natural and horticultural ecosystems at high risk of Phytophthora diseases. Forest Pathology, 46 (2), 134-163.

Orlikowski L.B., Ptaszek M., Rodziewicz A., Nechwatal J., Thinggaard K., Jung T. 2011. Phytophthora root and collar rot of mature Fraxinus excelsior in forest stands in Poland and Denmark. Forest Pathology, 41 (6), 510-519.

Vettraino A.M., Barzanti G.P., Bianco M.C., Ragazzi A., Capretti P., Paoletti E., Luisi N., Anselmi N., Vannini A. 2002. Occurrence of Phytophthora species in oak stands in Italy and their association 


\section{OPEN}

with declining oak trees. Forest Pathology, 32 (1), 19-28.

Trzewik A., Orlikowski L.B., Oszako T., Nowakowska J.A., Orlikowska T. 2015. The characterization of Phytophthora isolates obtained from diseased Alnus glutinosa in Poland. Baltic Forestry, 21 (1), 44-50.
White T.J., Bruns T., Lee S.J.W.T., Taylor J.L. 1990. Amplification and direct sequencing of fungal ribosomal RNA genes for phylogenetics. PCR protocols: a guide to methods and applications, 18 (1), 315-322. 